\title{
Manejo artroscópico en rupturas masivas del manguito rotador con espaciador subacromial
}

\author{
Arthroscopic management of massive rotator \\ cuff tears with subacromial spacer
}

\author{
René Ochoa Cázares, ${ }^{*}$ René Gabriel Narváez Corona, ${ }^{\ddagger}$ Alejandro González Parás\$
}

\section{Resumen}

Con los avances en cirugía artroscópica se ha logrado actualmente el manejo de lesiones masivas, sean reparables o irreparables, ofreciendo una mejoría importante en el dolor y la funcionalidad, y en ocasiones la fuerza muscular. Para elegir el tipo de tratamiento más adecuado debe tenerse en cuenta el síntoma principal, la edad, el grado funcional, la anatomía de la lesión y síntomas asociados. Entre las opciones terapéuticas se encuentran la debridación, la reparación parcial del manguito, la aumentación con parche, la reparación capsular superior y recientemente, la colocación de espaciador subacromial biodegradable. Éste último dispositivo es una adición importante al manejo en pacientes con tejidos deficientes, así como en la protección de reparaciones parciales.

Palabras clave: Lesiones masivas, manguito rotador, cirugía artroscópica.

\section{Abstract}

Arthroscopic surgery has reached a point where it has become a useful tool in the management of massive tears of the rotator cuff, reparable or not. This allows for an improvement in pain, functionality, and even muscular strength. Chief symptom, age, functional degree, lesion anatomy and associated symptoms must be evaluated to choose the best treatment. The available surgical options are debridement, partial rotator cuff repair, patch augmentation, superior capsule reconstruction and the use of a subacromial spacer. This option allows to improve shoulder function in patients with soft tissue compromise. It can also be used to protect partial repairs.

Keywords: Massive tears, rotator cuff, arthrospic surgery.

\section{INTRODUCCIÓN}

Las lesiones masivas del manguito rotador presentan un problema importante debido a que pueden comprometer la función del hombro en diferente medida. Actualmente contamos con diversos métodos de tratamiento por vía artroscópica que no comprometen la posibilidad de una segunda intervención, cuando ésta sea necesaria, ya sea nuevamente por vía artroscópica o abierta. A continuación, presentamos el caso de un paciente que fue reintervenido por una ruptura masiva del manguito rotador tras una reparación previa con anclas.

\section{CASO CLÍNICO}

Dr. René Gabriel Narváez Corona

Correo electrónico: narvaezrg87@gmail.com

Aceptado: 15-01-2020.

www.medigraphic.com/actamedica

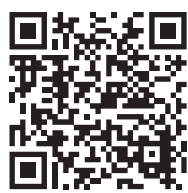

Paciente masculino de 60 años de edad acude a consulta por presentar dolor de tres semanas de evolución en hombro izquierdo así como limitación de los arcos de movilidad, 
principalmente para abducción y rotación externa. Lo asocia a ejercicio intenso en gimnasio. Un año antes había sido diagnosticado con ruptura de manguito rotador izquierdo, presentando la misma sintomatología. En esa ocasión fue manejado con reparación artroscópica de ruptura completa del supraespinoso hacia el margen bursal, por lo que requirió sutura con anclas. A la exploración física presenta dolor con los arcos de movilidad, con una elevación anterior de $80^{\circ}$, abducción de $70^{\circ}$ y rotación externa de $30^{\circ}$.

En resonancia magnética se reporta una ruptura completa del supraespinoso con retracción de la unión musculotendinosa que se sitúa sobre el margen superior de la cabeza humeral, de acuerdo con la clasificación de Patte corresponde a grado I. También se reporta artrosis acromioclavicular con formación osteocartilaginosa inferior y desplazamiento superior de la cabeza del húmero. No se reportan cambios artrósicos glenohumerales ni se observan en estudios radiográficos.

\section{Técnica quirúrgica}

Se programa para reparación del supraespinoso y tenodesis de la porción larga del bíceps, en la cual se realiza artroscopia de hombro izquierdo con posición de silla de playa. Se efectúa bursectomía subacromio subdeltoidea y sinovectomía, se observa ruptura completa transversal de las fibras del supraespinoso con retracción completa de sus fibras, y sin evidencia de desanclaje de las anclas empleadas en el procedimiento previo, dejando un espacio de $5.5 \mathrm{~cm}$. Se realiza reparación de la ruptura completa con suturas FiberWire mediante técnica side to side. Se verifica cierre

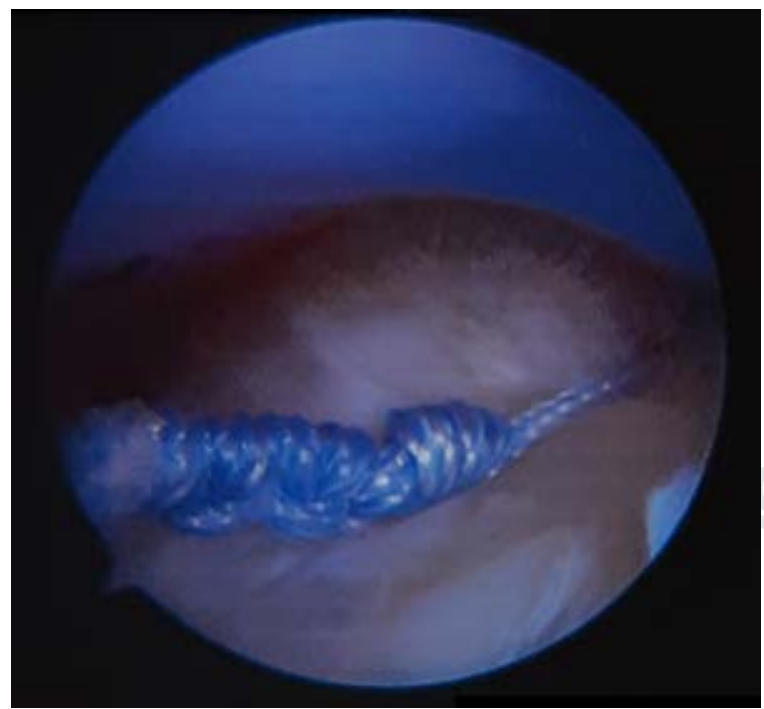

Figura 1: Suturas side to side para reparación de ruptura transversal de supraespinoso.

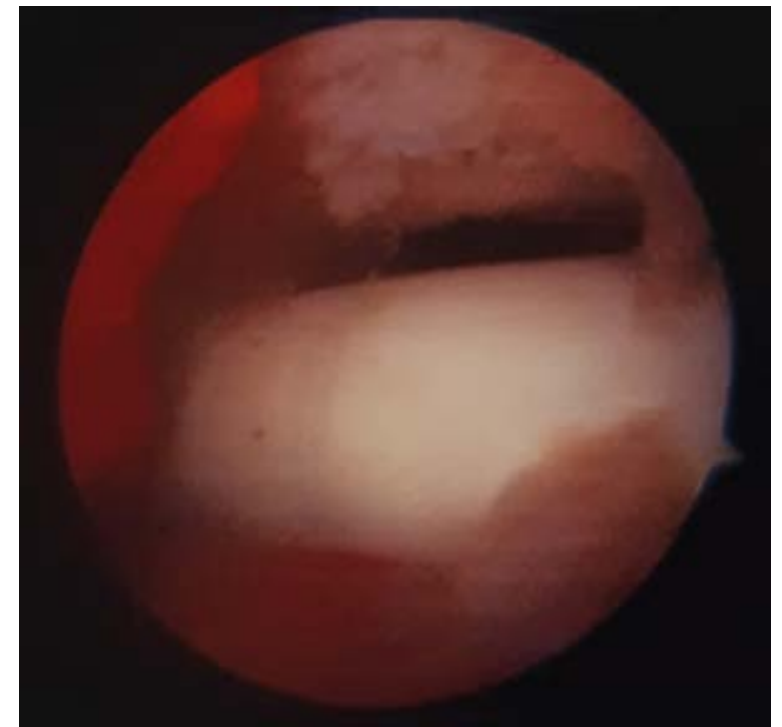

Figura 2: Introducción de cánula en espacio subacromial para colocación de InSpace ${ }^{\circledR}$.

de la sutura y se decide proteger reparación con espaciador subacromial InSpace. Su colocación se decide debido al grado de actividad tan intenso al que se asocia la ruptura de las fibras del supraespinoso en ambas ocasiones. Se introduce cánula de colocación con espaciador mediano $(50 \times 60 \mathrm{~mm})$ a través del portal lateral hacia el espacio subacromial luego de medir la distancia desde el borde lateral de la tuberosidad mayor hasta aproximadamente $1 \mathrm{~cm}$ medial al ápice glenoideo. Se realiza el inflado adecuado con $40 \mathrm{~cm}^{3}$ de solución salina para distender y se extraen $16 \mathrm{~cm}^{3}$ para la colocación final. Una vez optimizado por el rango de movimiento completo pasivo determinado, el volumen de inflado adecuado se dejó in situ al sellar el dispositivo utilizando el mecanismo que está integrado en el sistema de despliegue.

Una vez dado de alta se indica seguimiento en 10 días para retiro de puntos, e iniciar ejercicios pasivos de Codman. Posteriormente se envía a fisioterapia para mejorar arcos de movilidad y fuerza muscular. Actualmente el paciente presenta buena evolución con arcos de movilidad completos y sin dolor. Refiere sensación de disminución de la fuerza respecto a su otro hombro. Actualmente sus arcos de movilidad alcanzan $100^{\circ}$ de abducción, elevación anterior de $105^{\circ}$ y rotación externa de $40^{\circ}$, así como una fuerza muscular 5/5.

\section{DISCUSIÓN}

Las lesiones masivas de manguito rotador pueden llegar en ocasiones a ser irreparables por métodos convencionales. 
Se han descrito anteriormente como aquéllas que debido a su tamaño y retracción no pueden repararse en sus sitios de inserción de las tuberosidades a pesar de la movilización y liberación de tejidos blandos. ${ }^{1}$

Estos pacientes pueden presentarse con una variedad de síntomas, desde molestias leves hasta la limitación total de las actividades diarias y dolor severo. ${ }^{1}$ Otros síntomas asociados pueden ser el dolor por las noches, el cual interrumpe el sueño, e incluso pseudoparálisis. ${ }^{2}$ La pseudoparálisis se define como una flexión anterior activa $<90^{\circ} \mathrm{con}$ arcos de movilidad pasivos normales en ausencia de déficit neurológico. ${ }^{3}$ Clínicamente, el signo de Hornblower se ha descrito con una sensibilidad de $100 \%$ y una especificidad de $93 \%$ para identificar lesiones irreparables del redondo menor. El signo de retraso de rotación externa (lag sign) no permite vencer resistencia cuando se intenta la rotación interna o externa. ${ }^{1}$

De acuerdo a su tamaño, Bateman clasifica las lesiones masivas como grado IV, en la cual la lesión presenta un defecto de $>5 \mathrm{~cm}$. Ellman y Gartsman identificaron rupturas masivas como lesión de por lo menos tres tendones, o de dos tendones con retracción de $5 \mathrm{~cm} .^{2}$ DeOrio y Cofield clasificaron como rupturas masivas aquéllas que midieran $>5 \mathrm{~cm}$ en el sentido anteroposterior o mediolateral, y Gerber los describió como las que involucren al menos dos tendones. ${ }^{3}$

La resonancia magnética permite definir con mejor calidad los tejidos blandos, lo que permite identificar desgarros con 100\% de sensibilidad así como estimar hasta en $77 \%$ la anchura de una lesión, y retracciones hasta en

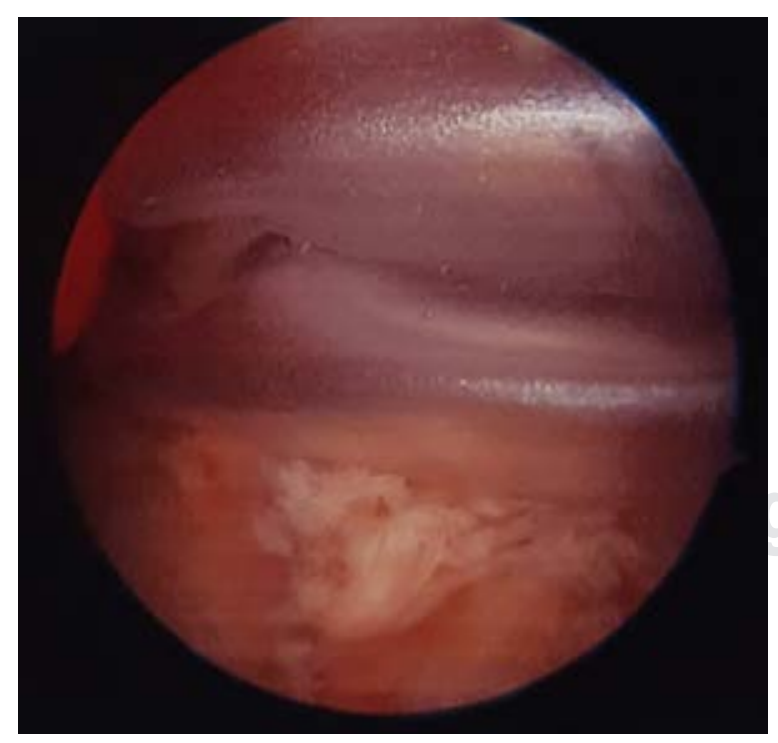

Figura 3: Llenado de espaciador subacromial InSpace ${ }^{\circledR}$ con solución salina.

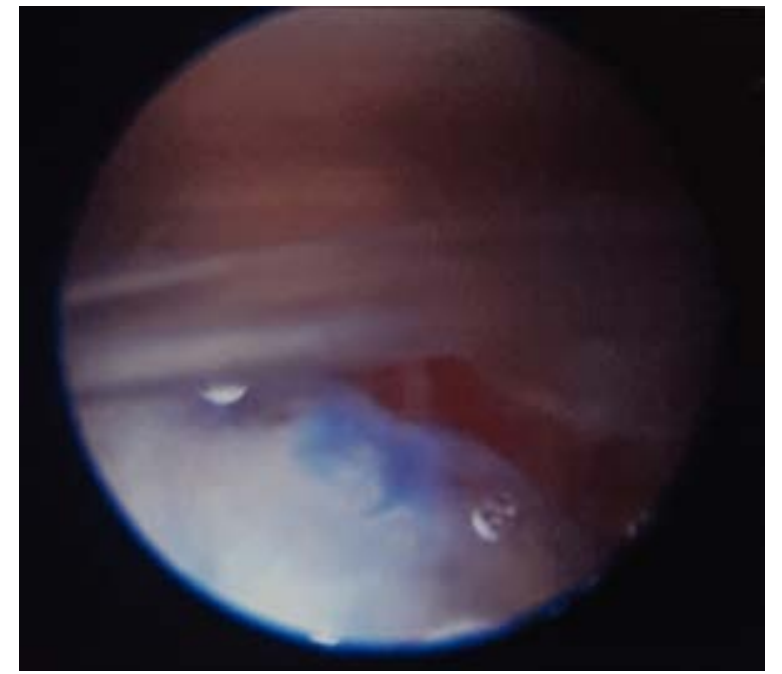

Figura 4: Posición final de espaciador. Se visualizan por debajo suturas para reparación de manguito rotador.

63\%. La cantidad de infiltración grasa está directamente asociada al riesgo de una rerruptura y a un pronóstico funcional malo.

El tipo de tratamiento depende de los síntomas, edad y nivel funcional del paciente. Además es importante un arco coracoacromial intacto, comorbilidades del paciente, y la presencia o ausencia de artrosis glenohumeral así como la estabilidad articular. ${ }^{1}$

Entre sus opciones quirúrgicas se incluye debridamiento subacromial con tenotomía del bíceps y acromioplastia con o sin reparación parcial, ${ }^{1}$ reparación completa, aumentación con parche, reconstrucción capsular superior. Otros procedimientos abiertos importantes en el manejo incluyen las reparaciones abiertas, la transferencia tendinosa y la artroplastia reversa de hombro. ${ }^{3}$

El espaciador subacromial es una novedosa técnica en la cual se emplea un espaciador biodegradable subacromial, éste se coloca entre el acromion y la cabeza humeral, el cual permite un deslizamiento suave y sin fricción, con lo que se restaura la biomecánica del hombro. ${ }^{4}$

El sistema consta de una cánula de colocación y un globo hecho de material biodegradable en un periodo de 12 meses, éste empieza a desinflarse a partir de los tres meses. ${ }^{4,5}$ Está contraindicado su uso en pacientes con alergia al componente L-láctido-co-e-caprolactona, infección latente o datos de necrosis tisular subacromial. ${ }^{4}$

Sus efectos de mejoría sintomática duran más que el periodo natural de degradación del implante. No se comprende por qué el dolor y la funcionalidad continúan mejorando tras el periodo de desintegración. ${ }^{4,5}$

Se recomienda su uso en pacientes con ruptura irreparable del manguito rotador sin artrosis glenohumeral tras 
seis meses de manejo conservador sin respuesta. Puede emplearse para proteger reparaciones parciales. ${ }^{4}$

Tras la reparación de rupturas masivas en las que el cirujano no está seguro de la calidad de la reparación o los tejidos blandos, puede ser complicado planear una rehabilitación que produzca resultados óptimos en el paciente, por lo que se ha optado por utilizar el espaciador subacromial como una protección para la fijación a corto y largo plazo. ${ }^{5}$

Sus riesgos incluyen respuesta a cuerpo extraño, irritación local del sitio de la herida, infección local, inflamación y necrosis tisular así como aumento del dolor y desplazamiento del componente. También se debe tener cuidado de no dañar el globo con el lente del artroscopio y no dejar espolones subacromiales que puedan dañar el dispositivo, e inflar el dispositivo adecuadamente para reducir el riesgo de luxación. ${ }^{4,5}$

Las recomendaciones para su colocación incluyen: realizar una descompresión adecuada del espacio subacromial; si existe duda entre el uso de dos medidas distintas, es preferible la más pequeña, ya que una sobrecobertura puede irritar el espacio subacromial. ${ }^{5}$

En una revisión sistemática realizada en 2018 se incluyeron exclusivamente lesiones masivas consideradas irreparables para manejo con este sistema, confirmadas durante la artroscopia, cuya sintomatología consistía en dolor persistente y pérdida de la función del hombro. La mayoría de los autores acordó la artrosis glenohumeral como criterio de exclusión, específicamente un grado > 3 de Hamada. La pseudoparálisis y las lesiones del subescapular también se consideraron contraindicaciones. Ésta última debido a posible migración anterior del espaciador. ${ }^{6}$

Los arcos de movilidad mejoraron considerablemente con elevación anterior de $124^{\circ} \pm 48^{\circ}$ a $147^{\circ} \pm 35^{\circ}$, abducción de $113^{\circ} \pm 53^{\circ}$ a $136^{\circ} \pm 43^{\circ}$, y rotación externa de $32^{\circ} \pm 19^{\circ}$ a $52^{\circ} \pm 25^{\circ}$. Hubo además una mejoría en la escala visual análoga de siete a 2.2 tras la cirugía. ${ }^{6}$

En un modelo experimental en cadáveres se comparó la efectividad del sistema InSpace contra la reconstrucción capsular superior mediante la ruptura de $5 \mathrm{~cm}$ de espesor completo del manguito rotador. Se encontró una migración de la cabeza humeral de $3.5 \pm 0.7 \mathrm{~mm}$ a $0^{\circ}$ de abducción, y de $2.9 \pm 0.6 \mathrm{~mm}$ a $30^{\circ}$ de abducción con ruptura completa del manguito rotador sin colocación de InSpace o reconstrucción capsular superior. A 60 y $90^{\circ}$ no se encontraron diferencias significativas comparadas con un hombro intacto. Tras la colocación del sistema InSpace se observó una traslación inferior de $2.8 \pm 1.9 \mathrm{~mm}$ respecto a su posición con la ruptura. La reparación capsular produce una traslación humeral inferior a $1.8 \pm 1.6 \mathrm{~mm}$ comparada con la ruptura. No se encontró diferencia significativa entre la posición sin ruptura y con el sistema InSpace bajo ninguna posición de abducción de hombro. Tampoco se encontró diferencia significativa entre el sistema InSpace y la reparación capsular superior. La fuerza de abducción también es significativamente inferior tras una ruptura de manguito rotador $(1.2 \pm 0.7 \mathrm{~N})$. Tanto el sistema InSpace como la reconstrucción capsular superior restauran la fuerza de abducción a un nivel que no fue estadísticamente significativo comparado con su estado intacto. ${ }^{7}$

Se estudiaron 15 casos entre 2014 y 2016, de los cuales tres habían tenido reparaciones fallidas previas. Al aplicar el test de Constant no se encontraron diferencias significativas a 12 meses, ni en el test QuickDASH. Los resultados mostraron una tasa de éxito de $53.3 \%$. No se logró determinar si existe algún grupo en específico de pacientes que se beneficie de este procedimiento respecto a rigidez, dolor como síntoma predominante o artrosis leve en estudios radiográficos. ${ }^{8}$

\section{CONCLUSIÓN}

La integración de procedimientos como el espaciador subacromial nos permite ampliar las opciones terapéuticas sin producir lesiones adicionales en los tejidos blandos preservando la articulación. Existen métodos adicionales no artroscópicos que pueden considerarse en caso de resultados no satisfactorios. La necesidad en un futuro de cirugías abiertas no se ve imposibilitada por la aplicación de las técnicas artroscópicas como el espaciador subacromial.

\section{REFERENCIAS}

1. Dines DM, Moynihan DP. Irreparable rotator cuff tears: what to do and when to do it; the surgeon's dilemma. J Bone Joint Surg Am. 2006; 88: 2294-2302.

2. Herrmann SJ, Izadpanah K. Tears of the rotator cuff. Causes-diagnosistreatment. Acta Chir Orthop Traumatol Cech. 2014; 81: 256-266.

3. Greenspoon JA, Petri M. Massive rotator cuff tears: pathomechanics, current treatment options, and clinical outcomes. J Shoulder Elbow Surg. 2015; 24 (9): 1493-1505.

4. Savarese E, Romeo R. New solution for massive, irreparable rotator cuff tears: the subacromial "biodegradable spacer". Arthrosc Tech. 2012; 1: e69-e74.

5. Bozkurt M, Akkaya M, Gursoy S, Isik C. Augmented fixation with biodegradable subacromial spacer after repair of massive rotator cuff tear. Arthrosc Tech. 2015; 4: e471-e474.

6. Moon AS, Patel HA, Ithurbun MP, Brabston EW, Ponce BA, Momaya AM. Subacromial spacer implantation for the treatment of massive irreparable rotator cuff tears: a systematic review. Arthroscopy. 2019; 35 (2): 607-614.

7. Singh S, Reeves J, Langohr D, Johnson JA, Athwal GS. The subacromial ballon spacer versus superior capsular reconstruction in the treatment of irreparable rotator cuff tears: a biomechanical assessment. Arthroscopy. 2019; 35 (2): 382-389.

8. Lorente A, Ruiz MA, Ruiz R, Vega, M, Álvarez R, Paniagua A, Díaz J. Malos resultados a corto plazo del balón subacromial InSpace ${ }^{\circledR}$. Resultados de 15 casos consecutivos con un año de seguimiento. Rev Esp Artrosc Cir Articul. 2017; 24: 197-203. 